\title{
L'efficacité rhétorique de Savonarole : l'exemple du premier sermon sur Amos et Zacharie
}

L'efficacia retorica di Savonarola: l'esempio del primo sermone su Amos

e Zaccaria

Savonarola's Rhetorical Efficiency: The Example of the First Sermon on Amos and Zechariah

\section{Cécile Terreaux-Scotto}

\section{OpenEdition} Journals

Édition électronique

URL : http://journals.openedition.org/cei/6138

DOI : $10.4000 /$ cei.6138

ISSN : 2260-779X

Éditeur

UGA Éditions/Université Grenoble Alpes

Édition imprimée

ISBN : 978-2-37747-159-1

ISSN : $1770-9571$

Référence électronique

Cécile Terreaux-Scotto, «L'efficacité rhétorique de Savonarole : I'exemple du premier sermon sur Amos et Zacharie », Cahiers d'études italiennes [En ligne], 29 | 2019, mis en ligne le 30 septembre 2019, consulté le 28 mars 2021. URL : http://journals.openedition.org/cei/6138 ; DOI : https://doi.org/ 10.4000/cei.6138

Ce document a été généré automatiquement le 28 mars 2021.

(c) ELLUG 


\title{
L'efficacité rhétorique de
} Savonarole : l'exemple du premier sermon sur Amos et Zacharie

\author{
L'efficacia retorica di Savonarola: l'esempio del primo sermone su Amos \\ e Zaccaria \\ Savonarola's Rhetorical Efficiency: The Example of the First Sermon on Amos \\ and Zechariah
}

Cécile Terreaux-Scotto

1 Dans son article sur «Michel Menot et la technique du sermon médiéval », Étienne Gilson remarquait qu'un sermon ne nous place pas «devant une œuvre littéraire à composer, mais devant une fonction religieuse à remplir » parce que la prédication vise à édifier l'homme ${ }^{1}$. Mais cette "fonction religieuse " implique de savoir convaincre pour avoir un effet immédiat. C'est dire combien la prédication est rhétorique : de l'art du prédicateur d'agencer ses arguments dépendra l'efficacité de son discours.

2 Cette question n'a pourtant été que rarement abordée dans l'immensité de la bibliographie concernant le dominicain Savonarole, où l'on trouve très peu d'éléments d'analyse de ses sermons à la lumière des artes prcedicandi, dont il dit d'ailleurs refuser les préconisations. Et si Savonarole affirmait ne pas suivre les règles de composition des sermons pour au contraire mieux les exploiter? Si, derrière ses revendications de "simplicité » et d' "immédiateté ", il y avait à l'inverse une recherche savante de la meilleure dispositio possible ? C'est à partir de cette hypothèse que j'ai entamé une recherche sur sa "mécanique du texte", pour reprendre la formule employée par Francis Goyet et Christine Noille dans leur présentation de la revue en ligne Exercices de rhétorique ${ }^{2}$.

3 Le texte savonarolien est bien particulier toutefois, puisque travailler sur la prédication implique de travailler sur des sources en partie insaisissables, ne serait-ce qu'en raison de leur caractère essentiellement oral. Marqués par la simultanéité de la communication et de la réception - ce que Paul Zumthor appelle la «culture de la 
voix »-, les sermons n'ont pas comme vocation première à constituer des textes écrits $^{3}$. De plus, la transcription écrite est nécessairement tributaire du talent du tachygraphe, des conditions matérielles de sa prise de notes et de ses choix (il peut juger inutile de restituer un passage, par exemple $)^{4}$. De façon symétrique, un sermon imprimé, y compris s'il bénéficie de la relecture du prédicateur, en porte justement l'empreinte ${ }^{5}$.

4 L'objet même de l'enquête est donc problématique ${ }^{6}$. En outre, les sermons écrits ne peuvent qu'être en deçà de la performance qui a eu lieu le jour où le sermon a été prononcé - il n'est que de songer à l'expérience spirituelle qui en est à la fois la source et le résultat - et par conséquent bien difficile à cerner, en dépit des témoignages des contemporains dont nous pouvons disposer parfois.

Cependant, cette première analyse rhétorique que j'entends mener ici étant destinée à tester une méthode d'investigation et à servir d'étalon pour l'étude de l'ensemble des sermons, il est fondamental que le texte retenu soit le plus proche possible de ce qu'on peut supposer qu'a dit Savonarole. Cela exclut dans un premier temps aussi bien les cycles sur Aggée et sur Job - établis par le notaire Lorenzo Violi à partir des autographes latins que Savonarole utilisait pour improviser ses sermons en langue vulgaire une fois qu'il était en chaire - que les cycles sur les Psaumes et sur Ézechiel, que le même Violi dit avoir consignés seulement «in substantia ». En revanche, Violi affirme avoir recueilli les sermons sur Amos et Zacharie, sur Ruth et Michée et sur l'Exode «dalla viva voce » de Savonarole.

6 Le notaire reconnaît toutefois les faiblesses de ses notes, « forse non così perfectamente et a punto come lui le ha predicate », en particulier, dit-il, en raison du flux des mots avec lequel une main, fût-elle "alata», ne peut pas rivaliser ${ }^{7}$. Mais ces trois cycles de sermons (Amos, Ruth, Exode) ont une particularité : ils ont été relus avant leur publication par Savonarole lui-même ${ }^{8}$, qui dans une lettre au pape Alexandre VI voit dans ces textes une transcription fidèle de ses propos'. Stefano Dall'Aglio a certes montré combien cette proclamation de «fidélité » (qui est d'ailleurs un topos chez les prédicateurs) ne suffisait pas à apporter la preuve que le texte écrit est la transcription exacte du sermon oral. D'un autre côté, puisque ces textes ont été approuvés par le prédicateur, il est légitime de les considérer conformes à la façon dont Savonarole envisageait l'art de prêcher. De plus, malgré les nombreux filtres par lesquels les textes étudiés sont passés, l'analyse ne peut être possible que si l'on estime qu'ils sont fidèles au prédicateur.

7 Pour délimiter le corpus, j'ai donc privilégié la fiabilité du texte plutôt que la chronologie des sermons - même si, comme nous le verrons, les circonstances constituent un élément d'interprétation déterminant pour la composition même du discours.

8 Par ailleurs, pour que l'enquête apporte des éléments probants, il est essentiel de comparer le dispositif savonarolien avec les sermons prononcés par les prédicateurs qui l'ont précédé. Parce qu'il a remporté autant de succès que Savonarole, Bernardin de Sienne peut constituer la base d'une comparaison fructueuse. Aussi ai-je choisi, parmi d'autres sermons possibles, un sermon de Savonarole articulé autour d'un verset que Bernardin avait lui aussi commenté. Il s'agit du premier sermon du cycle sur Amos et Zacharie bâti autour du verset 2 du psaume 39 («Dixi: custodiam vias meas ut non delinquam in lingua mea ») qui constituait le thème du neuvième sermon que Bernardin a prononcé à Sienne pendant le Carême de l'année 1427. 
Dans un premier temps, nous verrons que Savonarole exploite les circonstances dans lesquelles il prononce son sermon pour élaborer un plaidoyer prodomo. Nous analyserons ensuite comment il met l'exégèse biblique au service du temps présent pour mieux préparer l'avenir de Florence, dans un cadre où la parole du prédicateur et l'action politique ne font qu'un.

\section{La justification paradoxale du silence}

10 Un bref pontifical du 16 octobre 1495 avait confirmé à Savonarole l'interdiction de prêcher qu'il avait reçue le 8 septembre. Pourtant, le dominicain remonte en chaire le 17 février 1496 à la demande de la Seigneurie. L'autorisation écrite du pape, qui finit par céder aux autorités florentines et tolérer que le prédicateur reprenne ses fonctions, arrivera quelques jours plus tard à Florence. Dans un tel contexte, on pourrait s'attendre à ce que Savonarole justifie sa prise de parole, puisqu'elle va à l'encontre de l'autorité pontificale.

11 C'est pourtant en premier lieu à propos de son silence de l'automne 1495 qu'il va donner des explications - le dernier sermon qu'il a prononcé remonte au 22 octobre. Cette attitude paradoxale - expliquer un silence dont les causes étaient de notoriété publique - est particulièrement habile.

12 Justifier son silence permet à Savonarole de présenter a contrario sa prise de parole comme allant de soi, comme si prêcher relevait d'une telle évidence que le sujet ne valait même pas la peine d'être abordé. L'anomalie est du côté du silence, c'est de ne pas parler qui est une aberration, comme si le prédicateur ne se définissait que par la parole. C'est aussi une façon de dire que rien ni personne ne pourra l'empêcher de prêcher et de faire de sa seule présence en chaire un signe éloquent ${ }^{10}$.

13 Le premier moment du développement du sermon, de la dilatatio, consiste donc pour Savonarole à expliquer pourquoi il n'a pas parlé entre le 22 octobre 1495 et le 17 février 1496 , pourquoi il a tardé à entrer en lice ( indugiato a venire in campo $\left.{ }^{11} »\right)$.

14 Ce moment comprend trois arguments développés de façon très inégale, ne serait-ce que d'un point de vue quantitatif: le premier argument comporte quelques lignes quand le troisième ouvre sur un discours qui pourrait constituer à lui seul un deuxième sermon à part entière.

15 Le dispositif dominant est celui de la percontatio, une variante de la sermocinatio dialogique, qui consiste pour un orateur à imaginer un dialogue avec son adversaire ou son auditoire. Cette alternance de questions et de réponses, qui évoque un interrogatoire ${ }^{12}$, permet à Savonarole de prononcer un véritable auto-plaidoyer.

16 Il se défend tout d'abord d'avoir eu peur d'être assassiné. C'est pourtant bien une crainte de cet ordre qui avait motivé son refus de se rendre à Rome au début de l'automne. Dans sa lettre du 29 septembre 1495 au pape, le prédicateur évoquait en effet les "nemici capitali» et le "pericolo di vita ${ }^{13}$ " qui le menaçaient. Mais le 17 février 1496, l'évocation de ces dangers devient au contraire un argument pour nier sa peur : "Se io avessi avuto paura non saria venuto ancora adesso, perché io porto maggior periculo al presente che prima.»(p.8) Savonarole légitime alors son silence par une réponse sous-tendue par un raisonnement syllogistique : 
Majeure :Je n'ai pas peur aujourd'hui alors que je suis en danger.

Mineure : Or le danger est encore plus grand que par le passé.

Conclusion : Donc mon silence passé n'est pas dû à ma peur.

17 Le deuxième argument que Savonarole examine pour identifier les causes de son silence est centré sur la menace d'excommunication brandie par le pape. À la question « Hai tu dunque avuto scrupolo di conscienza di predicare? ", Savonarole feint la surprise. Sa réponse, par le biais d'une question faussement naïve («O perché? »), à laquelle ses interlocuteurs imaginaires répondent qu'ils ont ouï dire qu'il était excommunié, est l'embrayeur à partir duquel le prédicateur peut exposer les arguments de sa défense qui sont au nombre de trois.

Le premier argument prend la forme d'une parabole, qu'il avait déjà racontée le 11 octobre 1495 mais qui apparaît ici sous une forme bien plus développée ${ }^{14}$.

\begin{tabular}{|c|c|c|}
\hline $\begin{array}{l}\text { Prediche sopra } i \text { Salmi, } \\
\text { XXVIII, } 11 \text { octobre } 1495\end{array}$ & $\begin{array}{l}\text { Prediche sopra Amos e Zaccaria, I, } \\
17 \text { février } 1496 \text { (p. 9-10) }\end{array}$ & Commentaires \\
\hline $\begin{array}{l}\text { Ben sai che noi ubidiremo al } \\
\text { santo Padre [...] Io non dico } \\
\text { per questo che e' sia tratta } \\
\text { scomunica nessuna, ma la } \\
\text { cercano. }\end{array}$ & $\begin{array}{l}\text { non ti ricordi che io ti dissi che ancora che } \\
\text { la venisse [la scomunica] non varrebbe } \\
\text { nulla }\end{array}$ & $\begin{array}{l}\text { Contexte : Savonarole doit se } \\
\text { défendre contre une menace } \\
\text { d'excommunication à un } \\
\text { moment où il n'a pas le droit } \\
\text { de prêcher. }\end{array}$ \\
\hline Dimmi un poco & $\begin{array}{l}\text { te lo dimostrai per una parabola }[\ldots . .] \text { la } \\
\text { quale non mi rincrescerà a replicarla }[. . .]\end{array}$ & $\begin{array}{l}\text { Introduction de la parabole: } \\
\text { Savonarole rappelle qu'il l'a } \\
\text { déjà exposée quelques } \\
\text { semaines plus tôt, le } \\
11 \text { octobre } 1495 .\end{array}$ \\
\hline $\begin{array}{l}\text { tu hai uno figliuolo che sta } \\
\text { alla tua vigna a conciarla, }\end{array}$ & $\begin{array}{l}\text { Un cittadino aveva una bellissima vigna, la } \\
\text { quale faceva di molto frutto per operazione } \\
\text { e industria d'un suo buon figliuolo. }\end{array}$ & $\begin{array}{l}\text { La vigne est l'allégorie du } \\
\text { Royaume des Cieux, de } \\
\text { l'Église; le fils est le serviteur } \\
\text { de Dieu, en l'occurrence ici il } \\
\text { s'agit de Savonarole. }\end{array}$ \\
\hline
\end{tabular}




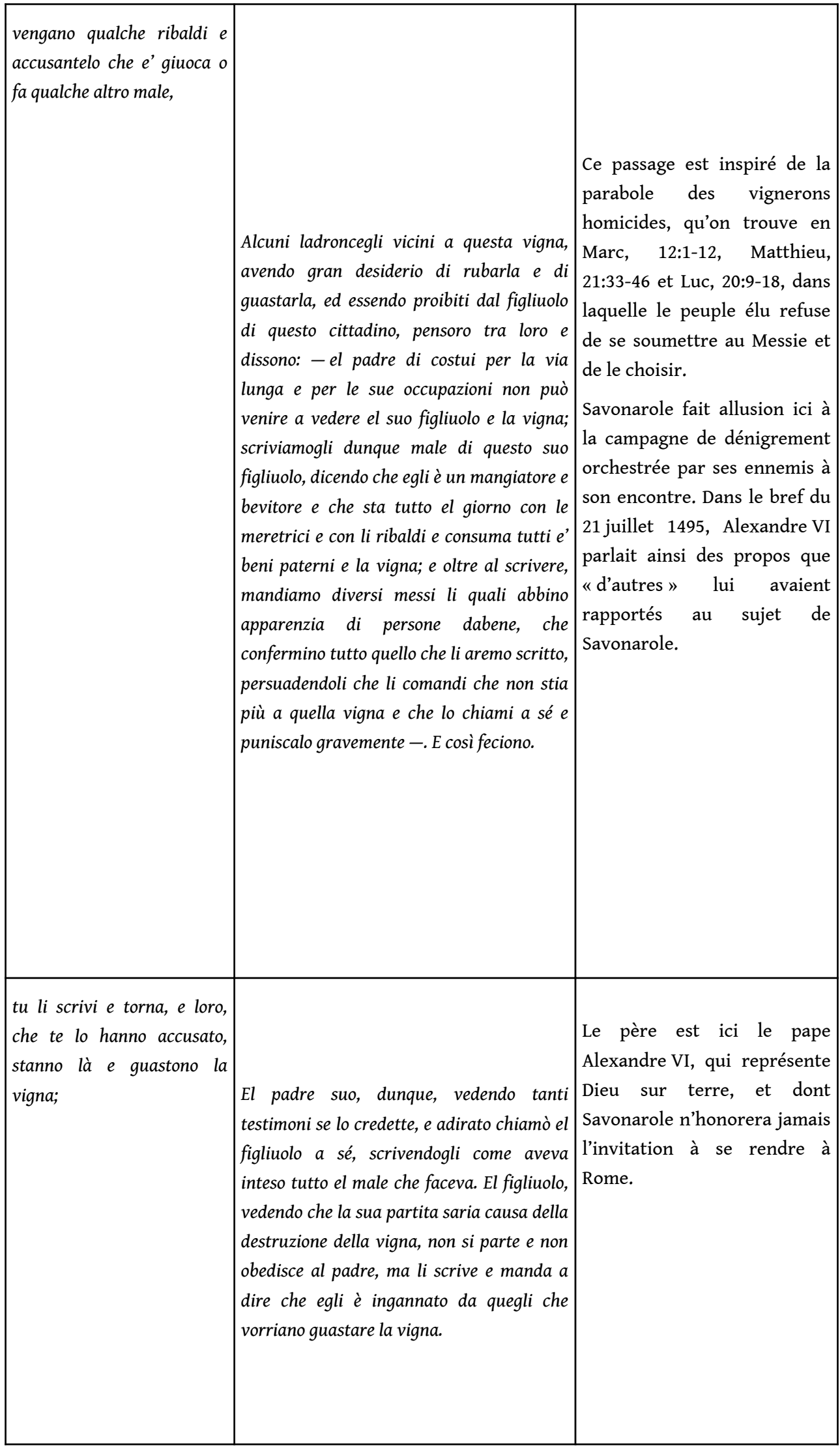




\begin{tabular}{|c|c|c|}
\hline $\begin{array}{l}\text { dimmi, quando il padre sa } \\
\text { poi il vero e che il figliuolo } \\
\text { non faceva male alcuno, non } \\
\text { credi tu che egli dica: }\end{array}$ & $\begin{array}{l}\text { Dimmi, cittadino: pare a te che questo } \\
\text { figliuolo abbi fatto bene o male, o che egli } \\
\text { abbi fatta la volontà o contro la volontà del } \\
\text { padre? }\end{array}$ & $\begin{array}{l}\text { L'interrogation sur le respect } \\
\text { de la volonté du père fait écho } \\
\text { à la parabole racontée en } \\
\text { Matthieu, 21:28-32. Le fils qui } \\
\text { fait la volonté de son père est } \\
\text { celui qui, après avoir désobéi, } \\
\text { se repentit et cultive la vigne, } \\
\text { à l'opposé de son frère, qui, } \\
\text { après avoir accepté de s'en } \\
\text { occuper, ne le fait pas. } \\
\text { En effet l'intention seule ne } \\
\text { suffit pas, encore faut-il agir. }\end{array}$ \\
\hline $\begin{array}{l}\text { - Pazzo che tu se', tu non } \\
\text { dovevi venire -. }\end{array}$ & $\begin{array}{l}\text { Certo, se tu non sei uno sciocco, tu } \\
\text { risponderai che egli ha fatto } \\
\text { prudentissimamente, non contra ma } \\
\text { secondo la volontà del padre. }\end{array}$ & $\begin{array}{l}\text { D'un point de vue littéral, } \\
\text { Savonarole loue sa propre } \\
\text { prudence qui a consisté à ne } \\
\text { pas se rendre à Rome. D'un } \\
\text { point de vue allégorique, il } \\
\text { nourrit la réflexion sur } \\
\text { l'obéissance et met en scène } \\
\text { l'épikie théorisée par Thomas } \\
\text { d'Aquin. }\end{array}$ \\
\hline
\end{tabular}

19 Une comparaison rapide de ces deux passages montre que la parabole racontée en 1495 ne contient que la charpente de la parabole de 1496. On ne peut pas exclure que cette différence soit due à une prise de notes de moindre qualité. Car lorsqu'en 1495 le notaire Lorenzo Violi prend en notes les sermons sur les Psaumes, il n'a encore jamais pratiqué ce type d'exercice ; il reconnaît lui-même ne pas avoir pris de notes « de verbo ad verbum, ma in substantia ${ }^{15}$ ". Le résultat de cette première tentative n'a en outre pas été relu par Savonarole, contrairement au texte du sermon sur Amos. C'est pourquoi une analyse des points communs aux deux textes (en particulier du sens général de l'argumentation et de sa tonalité) faisant fi des différences sera plus fructueuse qu'un essai de comparaison qui ne pourrait reposer que sur des hypothèses invérifiables (en ce qui concerne la spécificité du lexique par exemple).

À un niveau littéral, Savonarole s'appuie sur ce que la rhétorique appelle la preuve par les circonstances : le rôle qu'il entend jouer dans la cité, autrement dit les circonstances de temps, de lieu et de manière, servent d'argument pour justifier qu'il ne s'est pas rendu à Rome auprès du pape. Il se doit de rester en vie pour s'occuper de Florence. 
21 Mais à un niveau allégorique, le rôle de gardien de l'Église que s'attribue Savonarole introduit la question de la désobéissance. De la même façon que le fils de la parabole a bien agi en ne respectant pas la volonté de son père pour protéger sa vigne, le prédicateur agit de façon légitime en désobéissant au pape pour prêcher. Car comme l'a théorisé Thomas d'Aquin, l'épikie autorise à corriger la loi et à ne pas s'y conformer lorsque ses prescriptions à caractère universel deviennent défectueuses dans une situation particulière ${ }^{16}$. Autrement dit, il est permis de désobéir à une loi au nom de principes supérieurs.

Déjà en juillet 1495, Savonarole avait expliqué dans une lettre au pape Alexandre VI qu'étant donné que la réforme morale et politique de Florence voulue par Dieu exigeait sa présence dans la cité du lys, il ne pouvait pas partir à Rome sans porter préjudice au bien commun ${ }^{17}$. Le sens de la parabole qu'il raconte le 17 février 1496 n'est donc pas différent.

Mais il a recours à une parabole plutôt qu'à une argumentation théologique et juridique de type apodictique, c'est-à-dire nécessairement vraie, parce qu'il privilégie, dans ce sermon comme dans l'ensemble de sa prédication, un art de persuader dont l'efficacité repose sur une pédagogie par la maïeutique. Il attend de son auditoire qu'il arrive de lui-même à la conclusion qui s'impose, à savoir que sa désobéissance à Alexandre vi est légitime ${ }^{18}$. Pour faciliter la tâche à ses ouailles, il développe donc ensuite deux exempla, qui n'ont pas vocation à illustrer un argument, mais sont un «élément constitutif de l'argumentation du discours ${ }^{19}$ ".

24 Le premier exemplum expose ici une situation fictive, mais bien ancrée dans la société marchande à laquelle sont habitués les Florentins. À la manière de saint Augustin, qui se référait sans cesse aux "réalités [...] du monde environnant ${ }^{20}$ ", Savonarole fait de l'univers de référence de son auditoire un instrument de persuasion. Il demande à ses auditeurs de supposer qu'ils auraient confié une affaire à Bruges à un employé, et qu'une campagne de dénigrement serait menée par des concurrents jaloux contre ce même employé. Puis il imagine le comportement de chacun: le patron écrirait à l'employé pour le renvoyer sur la base de ce qu'il a entendu dire ; de son côté, l'employé reviendrait à Florence, négligeant du même coup l'affaire dont il a la charge à l'étranger. Savonarole déduit alors de ces informations ce que serait, ou plus exactement, ce que devrait être, le comportement du patron dans une situation réelle similaire. Il devrait bien sûr désapprouver le retour de son employée ${ }^{21}$.

À un niveau littéral, les Florentins-patrons sont les protagonistes d'une anecdote pédagogique fictive. Mais à un niveau allégorique, les Florentins se retrouvent dans la position du pape, amené à juger le comportement de son employé, autrement dit Savonarole. Le commerce à Bruges représente la chaire, que le prédicateur ne doit pas abandonner même si son patron - le pape - a accordé du crédit aux accusations portées contre lui et l'a renvoyé. D'une certaine façon, les Florentins se retrouvent ainsi juge et partie. Il y a là pour Savonarole une manière efficace de prendre à témoin son auditoire et de le contraindre, sinon malgré lui, du moins de façon passive, à adopter une attitude d'indulgence vis-à-vis d'un prédicateur désobéissant.

Le deuxième exemplum est tout aussi fictif, mais il est situé dans une dimension spatiotemporelle indéterminée. Le dominicain imagine qu'un serviteur œuvrant sous les ordres d'un baron commence à l'emporter sur les ennemis du roi. Dans un deuxième temps, le baron demanderait au serviteur de s'effacer, ce qui aurait pour effet de donner du champ aux ennemis du souverain. Mais le serviteur qui refuserait d'obéir au 
baron pour continuer à maîtriser les ennemis du roi et satisfaire de la sorte son souverain aurait raison. Du moins est-ce ainsi que Savonarole conclut l'anecdote, impliquant à nouveau son auditoire dans une prise de position favorable à sa propre personne. D'un point de vue allégorique, on peut en effet voir représentée ici la hiérarchie de l'Église, dont le serviteur le plus humble serait Savonarole qui, sous les ordres du baron-pape, protégerait son souverain, Dieu ou l'Église, de ses ennemis. Et puisque dans ce récit la position du pape est présentée comme allant à l'encontre des intérêts de l'Église, la désobéissance du prédicateur se trouve une nouvelle fois justifiée.

Or, si le prédicateur est fondé à être désobéissant, cela signifie qu'il a toute légitimité pour monter en chaire. Et s'il a toute légitimité pour monter en chaire, alors l'excommunication dont il est la cible n'est pas valable. Et si l'excommunication n'est pas valable, alors son silence n'était pas dû à l'excommunication. Là encore, Savonarole bâtit son discours sur un raisonnement de type syllogistique, dans lequel les propositions s'enchaînent de façon déductive.

Après avoir conclu le premier temps de sa démonstration en affirmant que son silence n'était dû ni à une raison passionnelle (la peur), ni à une raison d'ordre judiciaire (l'excommunication), Savonarole fait dire à son auditoire, toujours dans le cadre de la percontatio: "Che è stato dunque, frate? Tu ci tieni troppo a bada.» Il y a là une façon implicite de dire qu'il n'a pas encore abordé l'argument décisif. Mais il s'agit moins de souligner une défaillance que de suggérer, au contraire, qu'il maîtrise parfaitement le contenu de ce qu'il a à dire. Entretenant une sorte de suspens, il scande son propos en citant pour la troisième fois le verset biblique qui constitue le thème du sermon : « Dixi: custodiam vias meas ", à la fois pour reprendre le point de départ de son raisonnement et pour repartir comme d'ordinaire du latin, pour lequel il va faire office de traducteur improvisé.

Pour écarter la peur et les scrupules de ses motivations à garder le silence, Savonarole n'a pas construit un raisonnement théorique. Il est au contraire parti de sa situation réelle, quotidienne, de prédicateur excommunié et entouré d'ennemis menaçants. Il va faire de même pour le troisième argument qu'il examine. Il annonce en effet que, ayant observé combien sa parole avait suscité de contestation, il a fait son examen de conscience pour voir si elle n'était pas fautive. Or, il constate qu'il n'a commis aucune erreur intellectuelle quant à la philosophie, la foi ou la prophétie; il n'a commis aucun péché d'orgueil ou d'avarice; il n'a ressenti aucun désir coupable; il n'a éprouvé ni colère ni haine envers ses adversaires. Son silence n'est donc pas dû à une raison d'ordre moral.

En somme, Savonarole a donné trois démentis successifs. S'il ne prêchait plus, ce n'était ni par peur, ni à cause de la loi, ni par scrupule. Il peut ainsi vider de leur substance les charges qui pèsent contre lui. Car en plus de se présenter sous les traits de l'accusé, il s'est également fait son propre avocat. Aussi prend-il à parti aussi bien ses ennemis, qu'il désigne par la périphrase "tu che scrivi a Roma tante bugie", que son auditoire, désigné par le mot «cittadino" qui renvoie à la collectivité politique. Son habileté consiste à désamorcer, en les dénonçant par anticipation, les accusations de désobéissance envers le pape :

O tu che scrivi a Roma tante bugie, che scriverrai tu ora? Io so bene quello che tu scriverrai. - $O$ che, frate? - Tu scriverrai che io ho detto che non si debba obedire al Papa e che io non voglio obedire. Io non dico così. Scrivi come io ho detto e vedrai che non farà per te. (p. 11) 
31 Jouer le "rôle du naiif ${ }^{22}$ » dans un dialogue fictif de questions-réponses est un artifice récurrent de la langue homilétique, qui permet de conserver ou de réveiller l'attention de l'auditoire. Mais chez Savonarole ce dialogue est la forme la plus élaborée de la prolepse, qui consiste pour un locuteur à exposer les objections qui lui sont faites pour mieux les réfuter. Surtout, dans l'édifice savonarolien, la prolepse est bien autre chose qu'un ornement de la langue destiné à plaire à l'assemblée : elle est un moment clé particulièrement efficace de son discours de défense.

Premièrement, d'un point de vue éthique, dénoncer ce qu'il dit être la pensée de ses ennemis lui permet de montrer qu'il n'est pas dupe; c'est une façon de poser sa supériorité sur ses adversaires. Deuxièmement, d'un point de vue judiciaire, l'anticipation des critiques fonctionne comme un bouclier défensif à effet boomerang, qui lui permet de renvoyer ces mêmes critiques à ceux qui les ont faites. D'accusateurs qu'ils sont, les adversaires du prédicateur deviennent accusés. Letizia Pellegrini a montré comment les détracteurs de Savonarole utilisaient ses sermons pour l'accuser, quand dans le même temps le prédicateur les emploie de son côté pour se défendre ${ }^{23}$. Mais on peut ajouter que Savonarole fait lui-même de ses sermons le lieu de recueil des accusations dont il est victime, de façon à les récuser. Troisièmement, du point de vue de la construction du discours, ce dialogue fictif avec ses détracteurs crée un accord implicite entre le prédicateur-accusé et l'auditoire-procureur. Placés sur la même ligne que le prédicateur, les auditeurs deviennent d'une certaine façon ses complices, de sorte qu'ils ne peuvent plus conserver leur statut d'accusateurs. Et s'il n'y a plus d'accusateurs, le processus défensif peut pleinement s'accomplir.

Ce premier sermon sur Amos et Zacharie constitue donc un plaidoyer prodomo. De la même façon que Gisèle Mathieu-Castellani a pu parler de «la scène judiciaire de l'autobiographie », on pourrait parler pour Savonarole de "la scène judiciaire de la prédication ». Si l'espace qui m'est imparti ici ne me permet pas de développer la question de la contamination de la prédication par le genre judiciaire, elle mérite en tout cas d'être posée, car le dominicain aborde constamment la question de la légitimation et de la légitimité de sa parole.

\section{De l'actualité florentine à l'exégèse biblique}

Après avoir écarté toutes les raisons possibles de son silence et dit, en définitive, ce qui n'était pas, Savonarole explique pourquoi il a décidé de prêcher.

Il commence par citer le verset 2 du psaume 39 que nous avons déjà évoqué : «Dixi: custodiam vias meas ut non delinquam in lingua mea.» Il s'agit de ce qu'on appelle le « thème » du sermon, « fondement même de tout l'édifice oratoire ${ }^{24}$ ".

Cinquante ans plus tôt, Bernardin de Sienne avait choisi le même thème pour un de ses sermons de Carême. La structure de son discours était conforme aux règles du sermo modernus ${ }^{25}$. Après l'énoncé du verset biblique, d'abord en latin, puis en langue vulgaire - le thema -, suivait l'exorde qui présentait le sujet, commentait rapidement le verset biblique, et annonçait le plan qui serait suivi. Conformément aux recommandations des traités médiévaux, le thema déterminait la divisio du sermon :

Dixi: custodiam vias meas, ut non delinquam in lingua mea. [...] Unde di questo sacro parlare faremo tre considerazioni, di questa lingua. Primo, sarà considerazione, dove dice: «Dixi». Siconda sarà circumspezione, dove dice: «custodiam vias meas». Terza, conservazione, dove dice: «ut non delinquam in lingua mea». 

mot du thème constitue une étape du raisonnement, qui progresse par ramifications successives qu'on peut figurer sous la forme d'un arbre. Le verset-thème constitue les racines et le tronc, les parties de la division principale les grosses branches, les parties de la division secondaire les petites branches, et le développement, le feuillage ${ }^{27}$. Le sermon composé par Bernardin procède intégralement du thème. Si la conclusio a été négligée par le tachygraphe, la propositio énoncée clairement par Bernardin figure bien dans le texte écrit : «Stamane sarà da vedere il peccato che fa il detrattore, che per lo suo mal parlare fa contra la carità del prossimo. » De fait, le sermon de Bernardin s'apparente à une leçon de théologie. Nous n'avons connaissance que de la première partie de l'analyse - les deux autres parties, absentes, ont sans doute été perdues ou n'ont pas été prises en note, et il est plausible que la transcription écrite ait été lacunaire y compris pour le début du texte, dans la mesure où certains points, contrairement à d'autres, ne sont pas subdivisés. Malgré tout, nous pouvons voir qu'il s'agit d'analyser les méfaits de la médisance d'un point de vue très théorique, en répartissant la matière par énumération et par développements symétriques.

Frère Jérôme commence par expliquer longuement que Dieu, pour manifester sa bonté infinie, a créé une multitude de créatures. Il développe son argumentation dans le cadre de la sermocinatio, sous la forme d'un dialogue (colloquia personarum) composé de questions-réponses introduites par des formulations anaphoriques. Il imagine une question posée à son auditoire: "se tu fussi domandato", question qui commence invariablement par une élucidation : «che vuol dire che ». Son habileté consiste ensuite à créer un accord avec l'assemblée en lui attribuant la réponse: " tu risponderesti che ». Plutôt que de recevoir de façon passive une démonstration théorique, les auditeurs se trouvent ainsi impliqués dans un raisonnement qu'ils ne peuvent qu'approuver puisqu'ils sont mis dans la position d'être eux-mêmes les auteurs des réponses! La métaphore biblique du divin potier ${ }^{28}$ permet par ailleurs d'introduire un point central dans le sermon: s'il y a des vases précieux et des vases de mauvaise qualitéé ${ }^{29}$, c'est uniquement par la volonté du créateur. De la même façon, s'il y a des hommes bons et des hommes méchants, si certains sont prédestinés alors que d'autres sont condamnés, cela est dû à la volonté de Dieu, qui se manifeste de deux façons : la miséricorde pour les élus destinés à la vie éternelle, et la justice pour ceux qui sont réprouvés à cause de leurs péchés.

Après ce premier exposé théorique, Savonarole évoque la situation particulière de Florence. Citant pour la seconde fois le verset du trente-neuvième psaume, le dominicain annonce qu'il est remonté en chaire parce qu'il y a une multitude d'élus dans la cité. Il justifie alors sa désobéissance au pape par un raisonnement syllogistique destiné à convaincre du bien-fondé de sa décision :

Majeure : La prédication est faite pour les élus.

Mineure : Or il y a beaucoup d'élus à Florence.

Conclusion : Donc je vais prêcher à Florence.

41 C'est ce moment-là que Savonarole choisit pour exposer la divisio, qui partage le sermon en quatre étapes : après avoir exposé les raisons de son retard, il parlera à Dieu en son nom, puis au nom de son auditoire, avant de parler aux "méchants" et aux « incrédules». Chez Savonarole, la divisio ne découle donc pas du thema initial, qui 
constituait traditionnellement le centre de gravité du sermon, comme c'était le cas chez Bernardin de Sienne.

À propos de ce rôle central joué par le thema, Anne Régent-Sensini parle de la parole « anonyme et dépossédée » du prédicateur ${ }^{30}$. Or, Savonarole ordonne le développement de son sermon indépendamment du verset biblique qu'il commente, comme s'il devait affirmer son autorité de prédicateur. Il y a de fait chez lui une appropriation et une incarnation très forte de la parole divine, en particulier à travers les prédictions, la parole prophétique constituant un élément fondamental de l'édifice savonarolien ${ }^{31}$. Ainsi dit-il de façon emblématique : «Le mie parole procedono da Dio, le quali tu vedi che ogni giorno si vanno verificando. » (p. 46)

On peut en tout cas observer que l'auto-plaidoyer prend place après l'annonce du plan du sermon : une fois qu'il s'est posé en tant que prédicateur, Savonarole doit justifier sa prise de parole pour que le combat qu'il entend mener soit légitime. Pour traduire en langue vulgaire le début du verset biblique qui constitue le thema, Savonarole attend ainsi d'avoir fait son examen de conscience. Puisqu'il n'a décelé chez lui aucune faute intellectuelle ou morale, il a toute latitude pour s'exprimer. Mieux encore, il peut désormais faire l'exégèse du verset en même temps qu'il le cite intégralement. Il s'agit de :

- contrôler sa parole : «dixi: custodiam vias meas » = « io ho pensato da qui inanzi ancora di metterli buona guardia »;

- d'éviter le péché : «non delinquam in lingua mea » = "acciò che continuamente sieno monde non solamente da' peccati mortali, ma etiam [...] mi sforzerò che sieno monde e nette da' veniali »;

- de se surveiller : «Et ideo posui ori meo custodiam » = « io ho posto una guardia alla bocca mia »;

- toutes choses d'autant plus nécessaires que les pécheurs sont à l'affût pour lui nuire : «cum consisteret peccator adversum me » = « cioè stando sempre il peccatore contro a me ».

Après avoir justifié son silence, Savonarole explique donc pourquoi il a pris la parole. D'une position défensive, il passe à une position, sinon offensive, du moins active. Dans un premier temps, face aux injonctions contradictoires des «bons» qui attendaient qu'il prêche et des «mauvais » qui tentaient de l'en dissuader, il avait choisi le silence. Mais avec le temps, voyant que l'ardeur des uns diminuait tandis qu'augmentait la noirceur des autres, il était remonté en chaire, dans le but de "mettere divisione tra' buoni e li cattivi ».

Or, à partir du moment où Savonarole s'empare, pour ainsi dire, de la parole, tout se passe comme si un deuxième sermon commençait. Un deuxième verset biblique, toujours tiré du psaume 39, distribue désormais les différents moments du discours; c'est ce qu'on appelle un développement par "autorité concordante ${ }^{32}$ ». Savonarole parlera au Seigneur pour lui-même, puis aux justes, puis aux «mauvais ». Dans le premier passage, le prédicateur s'identifie au psalmiste qui se lamente en pensant à la brièveté de la vie avant de s'en remettre à Dieu; il se présente comme un homme trahi, moqué, poussé malgré lui à prendre la parole à Florence : "Avendoti già gran tempo pregato che tu mi donassi questa grazia che io non fussi mai obligato al governo d'altri, tu hai fatto tutto al contrario e mi hai tirato in questo loco a poco a poco. » (p. 19) Ce passage relève davantage de la prière personnelle que de l'homélie; de façon emblématique, le discours ne repose que sur les deux premiers pronoms du singulier, le premier pour le prédicateur et le deuxième pour le Seigneur. Il y a cependant, en toile de fond, le grondement des détracteurs : «[...] ogni uomo si fa beffe di me, perché già gran tempo io grido contro le iniquità e annunzio tribulazioni e vastità ed el parlar del signore è fatto a me in 
obbrobrio e in derisione tutto el dì. » (p. 20) Ces détracteurs seront les « mauvais » auxquels le prédicateur s'adressera à la fin du sermon.

Savonarole s'appuie ainsi sur l'exégèse biblique pour décrire sa propre situation. De façon générale, on observe que c'est moins l'exégèse biblique qui le conduit à parler de Florence, que Florence qui commande son exégèse biblique parce que, comme le souligne Gian Carlo Garfagnini, «la Bibbia è la sua chiave di lettura del mondo in cui si trova a vivere e che lo motiva nel suo agire ${ }^{33}$ ». De ce point de vue, il est particulièrement significatif que Savonarole ait envisagé la lecture de la Bible en fonction de la "diversité des temps", comme il l'expliquait en 1490 à un de ses détracteurs imaginaires qui lui reprochait de ne pas exposer la Bible à la manière des Anciens: "Cur non exponis ut antiqui? Sed tempus me cogit. Nam [...] variis modis pro tempore exposita est Scriptura. Ergo adhuc nostris temporibus et futuris variabitur ${ }^{34}$. " L'actualité est d'ailleurs toujours au cœur des sermons savonaroliens, comme l'ont illustré depuis longtemps les travaux de Jean-Louis Fournel et de Jean-Claude Zancarini en France et de Gian Carlo Garfagnini en Italie.

À l'échelle du premier sermon sur Amos qui nous occupe aujourd'hui, frère Jérôme passe systématiquement d'un discours théorique général (il y a des élus et des réprouvés) à un discours centré sur la ville de Florence (il y a beaucoup d'élus à Florence qui auront de plus en plus d'adversaires). À la fin du sermon, lorsqu'il reprendra le thème des élus et des réprouvés, il sera plus précis encore puisqu'il évoquera la division entre les fanciulli qui œuvrent pour la réforme voulue par Dieu et une partie des vieillards qui refusent ce projet exposé par lui.

C'est que conformément à un des piliers de sa prédication, Savonarole ne raisonne pas absolute, mais secundum quid ${ }^{35}$, autrement dit pas dans l'absolu, mais en fonction de la réalité florentine. Après avoir expliqué à l'assemblée des fidèles pourquoi les tribulations sont "justes et utiles", Savonarole en appelle donc à l'expérience des Florentins et évoque la guerre contre Pise :

E questo tu sai, popolo mio, non solamente per queste ragioni, ma etiam per esperienzia perché le tribulazioni che tu hai avute ti hanno convertito a Dio, e se tu consideri bene, Firenze, tu hai più guadagnato in questa avversità che tu non hai perso. Perché el vale più questo lume che tu hai acquistato, che non vagliono cento Pise. E quando tu non avessi mai ad avere altro da Dio, tu doverresti stare tacita e contenta, perché Pisa e tutti e' regni temporali passeranno, ma questo lume ti farà acquistare vita eterna. (p. 29-30)

Conquise par Florence en 1406, la ville de Pise avait profité de la chute du régime des Médicis et de la présence de Charles VIII en Toscane pour se rebeller. L'année 1495 avait été marquée par l'assaut manqué des Florentins emmenés par Paolo Vitelli contre la ville qui ne sera reconquise qu'en 1509. Or, Pise constitue un enjeu politique important dans le discours de Savonarole. Elle est en effet soutenue par les Français, à qui on sait que le prédicateur refuse de s'opposer puisque leurs armées sont selon lui guidées par le Christ. Aussi avait-il fait en sorte que Florence décline l'offre milanaise de faire partie de la ligue anti-française qui en mars 1495 rassemblait Venise, l'empereur Maximilien, le roi d'Espagne et le pape autour de Lodovico Sforza.

Savonarole utilise par conséquent ce conflit militaire et politique pour illustrer une question théologique - les tribulations sont justes et utiles. Mais cette question théologique nourrit à son tour des enjeux politiques. Pour le dominicain, les tribulations incarnées par l'armée de Charles VIII sont en effet nécessaires pour que l'Église se renouvelle, que les Florentins se convertissent, et qu'ils fassent la réforme 
politique que Dieu envisage pour Florence. Par ailleurs l'analyse, qu'elle soit théologique, philosophique ou politique, n'a pas de valeur en soi. Elle est toujours destinée à déboucher sur une proposition concrète, qui doit être appliquée dans un lieu précis - Florence -, à un moment précis de son histoire - celle du vide constitutionnel laissé par la chute de soixante ans de régime médicéen ininterrompu.

51 Dans ces conditions, la parole du prédicateur ne peut être envisagée qu'en tant qu'action politique.

\section{La prédication agissante}

52 Nous avons vu jusqu'à présent comment Savonarole a justifié son silence, puis motivé sa prise de parole. Il a exprimé combien la prédication s'imposait à lui, allant même jusqu'à en faire une question de survie : «Io vorrei star cheto e non parlare e non posso, perché il verbo di Dio è nel mio core come un foco, il quale se io nol mando fuora mi arde dentro le medulle e le osse. » (p. 20-21) Mais le fait est que quoi qu'il dise, au moment où il monte en chaire le 17 février 1496, le prédicateur brave une interdiction pontificale. Or, la désobéissance apparait précisément comme le véritable enjeu, la propositio du sermon, comme le suggère sa structure d'ensemble.

53 L'idée de prédestination constitue les fondations sur lesquelles repose le discours tout entier puisqu'elle distribue à l'intérieur du sermon la parole de Savonarole, qui s'adresse d'abord aux « justes », puis aux « mauvais ».

54 Aux «justes », Savonarole indique que Dieu a préparé la béatitude éternelle pour les "élus », ainsi que les moyens pour y parvenir. Parmi ces moyens figurent la prière et la prédication, qui ont déjà porté leurs fruits à Florence. Le dominicain s'attarde aussi longuement sur la transformation que les fanciulli, âgés de six à vingt-quatre ans, ont apporté dans la cité. Ils se sont réformés, faisant du Carnaval un Carême, apportant l'aumône là où auparavant ils pratiquaient l'extorsion, transformant les rires transgressifs en louanges divines. Il s'agit de l'œuvre de Dieu, affirme Savonarole, pour trois raisons : parce qu'un " fraticello » comme lui a réussi là où les autorités florentines ont échoué, parce que les enfants se sont attirés beaucoup d'ennemis et parce que les prédictions qu'il avait formulées se sont réalisées. Mais les enfants ne se sont pas réformés seulement d'un point de vue moral. Ils ont aussi fait un bon choix politique, puisqu'ils ont élu le Christ roi de Florence.

C'est justement ce contre quoi s'élèvent les « mauvais citoyens» (p.39). Le prédicateur présente alors les «mauvais» en opposition aux «justes", alternant les «voi» accusateurs et les «loro» de louange : "Voi amate la carne vostra e loro amano el spirito vostro; voi desiderate le cose temporali e loro vi desiderano le eterne; voi non pregate mai per voi medesimi e loro pregano continuamente per voi.» (p. 42) Or, si les « justes» sont aussi les «élus » de Dieu, les « mauvais » sont aussi les détracteurs de Savonarole. Ce qui signifie que les fanciulli et le prédicateur ont les mêmes ennemis. Mais tous savent résister à leurs adversaires: de la même façon que Savonarole parvient à tenir tête à ses détracteurs, les «élus » font face aux tribulations et à la « malizia » croissante de leurs ennemis. Dans ce combat, le prédicateur fait office de guide : «Io son mandato a questa volta per capitaneo, benché insufficiente e indegno. » (p. 6) Dans ces conditions, se trouver sur la chaire est déjà en soi un moyen d'agir contre ceux qui sont hostiles à la réforme. 

de la même façon que le prédicateur désobéit au pape en continuant à prêcher, les enfants refusent de prendre le «mauvais pli » du régime tyrannique, formulation qui désigne implicitement le régime médicéen : "Non aranno presa la piega del ciambellotto, come hanno presa i padri loro, che non si possono spiccare dal reggimento tirannico né sanno conoscere quanta è grande questa grazia della libertà. »(p. 38) À l'inverse, une partie de leurs aînés, que Savonarole appelle les "vecchi cattivi", refusent d'œuvrer pour le projet que Dieu a pour Florence : «Questi vecchi hanno fatto la piega del ciambellotto e non vogliono emendarsi de' loro vizii ${ }^{36}$."

Savonarole a ouvert son sermon avec l'idée que la prédestination distingue les " élus » des «mauvais ». Il le termine de façon symétrique sur l'image d'une Florence divisée. D'un côté se trouvent les vieux « scélérats ». Ce sont ses adversaires. Ils sont apparus à intervalles réguliers dans le sermon : ce sont les «cattivi», les « ministri di Satanas». Ce sont aussi ceux qui écrivent à Rome pour raconter des mensonges et lui nuire. Ce sont des « capassoni », des vauriens, des «ignoranti » qui parlent de choses qui les dépassent : "Volete disputare di cose che voi non intendete [...]. » (p. 43) L'emploi du verbe "disputare », qui renvoie aux débats théologiques, n'est évidemment pas innocent et suggère de façon ironique que les adversaires de Savonarole ne sont pas à la hauteur des enjeux. En face des «mauvais » se trouvent les jeunes générations, garçons et filles, mais aussi les bons citoyens et les femmes de bien, qui de leur côté ont tous choisi le bien vivre, à la fois condition et conséquence de la réforme politique. Savonarole adresse des menaces aux premiers: "Dio ti ritroverrà e punirà l'anima tua diabolica» et des encouragements aux seconds : "Attendete a crescere e perseverare nel ben vivere e pregate Dio che presto ci liberi da questa tribulazione [...]. » (p. 48-49)

Savonarole construit donc son sermon de façon circulaire et en spirale. Le discours n'est pas organisé par ramifications, mais par strates superposées qui s'alimentent par enchaînements successifs. Sans doute est-ce cela que voulait dire le chroniqueur Bartolomeo Cerretani lorsqu'il observait que Savonarole « introdusse quasi nuovo modo di pronunziare il verbo d'Iddio, cioè all'apostolesca, sanza dividere el sermone ${ }^{37}$ ». De chaque argument découle un autre argument qui en suscite un troisième qui à son tour en induit un quatrième qui va déterminer un cinquième argument et ainsi de suite : tout se passe comme si les arguments s'inscrivaient dans une démonstration dont l'enjeu fondamental serait la causalité ${ }^{38}$. Nous avons fait allusion plus haut à l'importance pour Savonarole d'agir selon la "diversité des temps»-que Machiavel appellera la "qualité des temps ». Mon hypothèse est que la situation inédite provoquée par l'état de guerre incessant après la descente de Charles VIII dans la péninsule entraîne chez Savonarole une modification de l'art traditionnel de prêcher; seule une comparaison des sermons prononcés avant et après 1494 permettra cependant de vérifier (ou d'invalider) cette interprétation ${ }^{39}$.

59 Par ailleurs, en terminant sur la figure de ceux qui choisissent d'obéir au Christ plus qu'à toute autre autorité, Savonarole légitime la désobéissance. Car, si les «mauvais » usurpent leur autorité en ne voulant pas « se détacher du gouvernement tyrannique ", ceux qui se convertissent au bien vivre et au bien gouverner sans leur prêter attention ont raison de ne pas leur obéir. En conclusion de son auto-plaidoyer, frère Jérôme avait d'ailleurs cité les Actes des Apôtres $(5,29)$ pour inviter à obéir à Dieu plus qu'à quiconque. 
En bravant l'interdiction pontificale de parler, Savonarole se présente par conséquent comme un modèle à imiter: il incarne la désobéissance. Le fait qu'il s'agisse d'une action dans laquelle le moyen - parler depuis la chaire - se confond avec la fin - désobéir au pape - la rend d'ailleurs particulièrement efficace. Au lieu de multiplier les exhortations morales à désobéir, Savonarole est ce qu'il pourrait dire de faire. Il se conforme en cela à la culture dominicaine pour laquelle l'exemplum a une fonction d'imago agens, d'image agissante. Les hommes " sono molto più mossi e tirati dalli esempli delle cose che non sono da ragioni o da parole ", constatera-t-il sur les traces de saint Grégoire dans le quatrième sermon sur l'Exode $e^{40}$. Mais ici Savonarole ne raconte pas un récit exemplaire : il est lui-même le récit exemplaire. Il agit en cela à la manière du Christ et de ses saints, qui contrairement aux « savants » qui n'enseignent que par les « mots », enseignent par les « faits » et les « œuvres ${ }^{41} »$.

En outre, la présence du verbe du Christ dans son corps («Cristo è quello che prèdica e non $i 0^{42}$ ", dit-il à plusieurs reprises) fait de lui une "figure exemplaire du témoignage devant la communauté des fidèles du Christ $^{43}$ ». Si bien que le simple fait de se faire voir avant même de se faire entendre a une valeur agissante.

Alors que frère Jérôme n'a de cesse de dire qu'il n'est que le porte-parole de Dieu, c'est en fait souvent sa propre expérience qui détermine l'argumentation d'un sermon tout entier. Ce premier sermon sur Amos en est un exemple frappant. On peut dire qu'en agissant le prédicateur formule un énoncé, mais que de façon symétrique c'est en parlant qu'il agit. Action rhétorique et action politique seront donc une seule et même chose.

Emblématique à cet égard est le statut des citations bibliques.

Dans le psaume 39, le locuteur expose la souffrance qui découle de sa décision de garder le silence; une souffrance tellement insupportable qu'il ne peut continuer à se taire. Choisir comme thème de son sermon l'extrait d'un texte qui est le drame du silence, c'est donc pour Savonarole agir en faveur de sa propre cause.

Plus largement, comme le formule Roberto Ridolfi, chez Savonarole «il Testamento Vecchio [...] diventava sulle sue labbra e negli orecchi dei suoi uditori una cosa attuale, non più l'arca di un remotissimo passato, ma lo specchio del presente, la chiave del futuro ${ }^{44}$ ". La façon dont le prédicateur évoque le temps biblique, le temps de Florence et le temps prophétique est en effet semblable à un tissage. Si l'on prend comme échantillon révélateur le premier moment du sermon d'ouverture du cycle sur Amos, on voit que la parole du prédicateur, prononcée dans la République florentine à la toute fin du $\mathrm{XV}^{\mathrm{e}}$ siècle, est la navette d'un métier qui noue inlassablement le fil de chaîne biblique au fil de trame prophétique :

\begin{tabular}{|c|c|c|}
\hline $\begin{array}{c}\text { Fil de chaîne. } \\
\text { Il s'agit d'une citation de la Bible } \\
\text { que Savonarole traduit en langue vulgaire ; } \\
\text { dans cette colonne je fais figurer le verset } \\
\text { extrait de la Bible. }\end{array}$ & $\begin{array}{c}\text { La navette. } \\
\text { Ici figurent les } \\
\text { commentaires de } \\
\text { Savonarole, que je } \\
\text { donne sous forme de } \\
\text { paraphrase. }\end{array}$ & $\begin{array}{c}\text { Fil de trame. } \\
\text { Ici sont indiquées sous } \\
\text { forme de paraphrase les } \\
\text { promesses que Savonarole } \\
\text { fait au peuple qu'il } \\
\text { pense élu. }\end{array}$ \\
\hline
\end{tabular}




\begin{tabular}{|c|c|c|}
\hline $\begin{array}{l}\text { Scimus quoniam diligentibus Deum omnia } \\
\text { cooperantur in bonum his qui secundum } \\
\text { propositum vocati sunt sancti. Romains, 8:28: } \\
\text { « Nous savons d'ailleurs que Dieu fait tout } \\
\text { concourir au bien de ceux qui l'aiment, ceux } \\
\text { qui sont appelés conformément à son } \\
\text { dessein.» }\end{array}$ & $\begin{array}{l}\text { La prédication du } \\
\text { verbe de Dieu est faite } \\
\text { pour le salut de ceux } \\
\text { qui sont prédestinés. }\end{array}$ & $\begin{array}{l}\text { Je vois qu'il y a beaucoup } \\
\text { d'élus à Florence. }\end{array}$ \\
\hline $\begin{array}{l}\text { Omnes qui pie vivere volunt in Christo Iesu, } \\
\text { persecutionem patientur. } 2 \text { Timothée, 3:12: } \\
\text { "Aussi bien tous ceux qui veulent vivre } \\
\text { pieusement en Christ Jésus seront } \\
\text { persécutés.» }\end{array}$ & $\begin{array}{l}\text { Je suis revenu ce matin } \\
\text { sur le terrain pour les } \\
\text { rassembler et les } \\
\text { consoler. }\end{array}$ & $\begin{array}{l}\text { Je vais chanter un psaume } \\
\text { Dixi custodiam vias meas } \\
\text { qui est destiné aux élus. }\end{array}$ \\
\hline $\begin{array}{l}\text { In finem pro Idithum canticum David. Ps, 31:1: } \\
\text { « Au maître de chant. De Yedoutoun.» }\end{array}$ & $\begin{array}{l}\text { Ce psaume est fait } \\
\text { pour les élus de Dieu. }\end{array}$ & $\begin{array}{l}\text { Ce psaume les conduira à } \\
\text { la vie éternelle. }\end{array}$ \\
\hline $\begin{array}{l}\text { Pax vobis: ego sum, nolite timere. Luc, } 24: 36: \text { «a } \\
\text { paix soit avec vous: c'est moi, ne craignez } \\
\text { rien.» }\end{array}$ & $\begin{array}{l}\text { Le Seigneur vous salue } \\
\text { ce matin. }\end{array}$ & $\begin{array}{l}\text { Pour vous consoler des } \\
\text { tribulations et des } \\
\text { adversités que vous } \\
\text { recevez des méchants } \\
\text { ministres de Satanas. }\end{array}$ \\
\hline $\begin{array}{l}\text { Non veni pacem mittere in terram, sed gladium. } \\
\text { Luc, 12:51: "Pensez-vous que je sois paru } \\
\text { pour donner la paix sur la terre? Non, je vous } \\
\text { le dis, mais la division." }\end{array}$ & $\begin{array}{l}\text { Mais notez de quelle } \\
\text { paix il s'agit. }\end{array}$ & $\begin{array}{l}\text { Il vous envoie la paix du } \\
\text { Seigneur. }\end{array}$ \\
\hline $\begin{array}{l}\text { Pacem meam do vobis, pacem relinquo vobis, non } \\
\text { quomodo mundus dat ego do vobis. Jean, 14:27: } \\
\text { "Je vous laisse la paix, c'est ma paix que je } \\
\text { vous donne; ce n'est pas comme le monde la } \\
\text { donne que moi je vous la donne.» }\end{array}$ & Je suis votre capitaine. & $\begin{array}{l}\text { Nous sommes là pour } \\
\text { combattre les démons, } \\
\text { nous serons victorieux. } \\
\text { Plus il y aura d'ennemis, } \\
\text { plus votre rédemption } \\
\text { sera proche. }\end{array}$ \\
\hline
\end{tabular}

À la lecture de ce tableau, il apparaît que non seulement il y a une cohérence entre toutes les citations de la Bible, qui pourraient se suffire à elles-mêmes, mais aussi que chaque passage est interprété en fonction d'une prédiction qui met en jeu l'avenir des Florentins. En reliant le temps biblique au temps prophétique, Savonarole construit ainsi progressivement une nouvelle Jérusalem, dans laquelle seuls les élus dirigent dans le sens voulu par Dieu. La citation de l'Ancien Testament relève donc de l'énoncé performatif au sens où le définit John Langshaw Austin ${ }^{45}$. Énoncer la Bible, c'est pour Savonarole bâtir les fondations d'une cité renouvelée; interpréter la Bible, c'est brandir la cible que les Florentins doivent atteindre. Le dominicain remarque d'ailleurs 
à plusieurs reprises que citer la Bible, c'est agir. Dès le 25 décembre 1494, il affirme que "le Scritture del Signore sono quelle che fanno ogni $\cos ^{46}$ ». Et le 17 février 1496, au cours de la prière qui clôt le discours qu'il adresse aux «élus », il supplie le Seigneur de parler : "Ah, Signore, ne sileas, non star cheto, parla, Signore, perché il tuo dire è fare, come è scritto: Dixit et facta sunt; se tu adunque di', saranno fatti tutti questi beni. »

Cette performativité de la parole divine (mais qui est aussi la sienne, puisqu'il se présente en tant que prophète) constitue même l'axe autour duquel Savonarole répond à la dernière accusation qui figure dans le premier sermon sur Amos : celle de «semer le trouble » dans la péninsule : "molti dicono che io ho conturbata la Italia ». Après s'être défendu de bénéficier d'une armée et d'ententes secrètes avec des dirigeants, il répond que les troubles auront et ont déjà lieu, mais qu'ils sont et seront dus à la guerre, à la peste et à la famine. Il part ensuite de l'accusation qui lui est faite en soulignant que sa parole ne peut pas être tenue responsable des désordres puisqu'il disait déjà la même chose quelques années auparavant, alors que les troubles n'avaient pas commencé. Et si les troubles n'avaient pas commencé, c'est parce que les "gens venus d'ailleurs " n'étaient pas encore venus pour perturber l'Italie. À l'issue de ce premier moment, Savonarole nomme les responsables des troubles: ce sont les Français, qui ont franchi les Alpes en septembre 1494. Frère Jérôme s'appuie donc ici sur un argument véridique. Pour établir la vérité, il met les accusations dont il est l'objet à l'épreuve des faits, en s'appuyant sur des connaissances notoires.

Mais frère Jérôme ne se contente pas de se disculper. Dans un deuxième temps, il parvient également à transformer l'accusation qu'on lui porte - semer le trouble - en éloge. Si on l'accuse de semer le trouble par sa seule prédication, dit-il en effet, alors c'est que sa parole est action. Puisqu'il s'agit là d'une propriété divine, mais qu'il n'est lui-même pas Dieu, il s'ensuit que ses paroles, en revanche, viennent bien de Dieu. Savonarole donne dès lors une pleine légitimité à sa parole, d'autant que les troubles que subissent l'Italie constituent à ses yeux une preuve de la nature prophétique de son discours. Par ailleurs, si ce que le prédicateur prédit vient de Dieu, et que le « dire » de Dieu est un «faire ", alors la parole du prédicateur est elle-même agissante.

\section{Conclusion}

$\mathrm{Au}$ terme de cette première analyse rhétorique des sermons de Savonarole, il apparait que nous sommes bien devant une œuvre dont nous pouvons analyser la composition par un prédicateur qui maîtrise parfaitement l'art rhétorique qu'il affirme pourtant refuser. L'analyse rhétorique du dispositif qu'il met en place pour remporter l'adhésion de son auditoire peut permettre de comprendre les raisons de son succès comme de sa chute. Mais la rhétorique nous amène alors à prendre en compte bien d'autres aspects.

Un élément essentiel, nous l'avons évoqué, consiste à mesurer si à la descente de Charles VIII dans la péninsule correspond un changement dans la construction des sermons. Une comparaison attentive de la composition des sermons qui ont été pris en note par Lorenzo Violi et de ceux qui ne sont que l'amplification ou la traduction plus ou moins sommaire et arbitraire des schémas latins conçus par Savonarole est à cet égard indispensable. Il convient également de s'interroger sur l'importance des destinataires: la composition de l'assemblée (Savonarole affectionne les sermons ad status) a-t-elle une influence sur l'agencement des arguments? Il est également impossible de faire l'économie d'une comparaison avec Bernardin de Sienne, mais aussi 
avec d'autres prédicateurs, antérieurs ou postérieurs à Savonarole, pour déterminer dans quelle mesure les questions formelles sont liées aux circonstances dans lesquelles les sermons, textes d'intervention, sont prononcés. Il me semble par ailleurs important de raisonner par cycles de sermons et d'accorder une place essentielle aux sermons d'ouverture et de fermeture de chacun des cycles, pour vérifier l'hypothèse, entrevue ici, d'une structure en spirale, faite de ressassements, de redites et d'approfondissements.

Ces strates successives sont élaborées au gré des événements que Savonarole choisit de commenter. Or, ces événements ne sont pas seulement politiques; ils sont aussi autobiographiques. C'est que Savonarole, qui comme on le sait se pense prophète et attend le martyre, incarne véritablement la parole : celle de la Bible, mais aussi celle qu'il élabore pour convaincre de la nécessité de la réforme morale, religieuse et politique. Nous avons donc à faire à la figure très forte d'un orateur se mettant en scène pour entraîner une cité tout entière à sa suite.

\section{BIBLIOGRAPHIE}

AcCAdEMIA D'OROPA, Alessandro vi e Savonarola (Brevi e lettere), Turin, ITER, 1950.

Austin John Langschaw, Quand dire, c'est faire, Paris, Seuil, 1970.

CHARLAND Thomas-Marie, o. p., Artes prcedicandi. Contribution à l'histoire de la rhétorique au Moyen Âge, Paris, Ottawa, J. Vrin, Institut d'études médiévales, 1936.

CONNESSON Anne-Laure, «L'apologue dans la prédication de Savonarole (Prediche sopra Amose Zaccaria, 1496) », Chroniques italiennes, $\mathrm{n}^{\text {os }} 2-3,2003$, p. 93-108.

DALL'AGLIO Stefano, «'Faithful to the Spoken Word': Sermons from Orality to Writing in Early Modern Italy », The Italianist, vol. 34, n 3, 2014, p. 463-477.

FOURNEL Jean-Louis, « Les temps de la prophétie dans la Florence savonarolienne (automne 1494été 1495) », dans A. Redondo (dir.), La prophétie comme arme de guerre des pouvoirs (XVe-XVII siècles), Paris, Presses de la Sorbonne Nouvelle, 2000, p. 191-202.

GARFAGNINI Gian Carlo, «Lorenzo Violi e il Savonarola », Introduction à L. Violi, Le Giornate, G. C. Garfagnini (éd.), Florence, Leo S. Olschki, 1986, p. IX-LXI.

GARFAGNINI Gian Carlo, "Questa è la terra tua». Savonarola a Firenze, Florence, Sismel Edizioni del Galluzzo, 2000.

GARFAGNINI Gian Carlo, " Dire tutto quello che nel libro si contiene. La Bibbia di Savonarola », Archa Verbi, vol. 6, 2009, p. 121-132.

GiLSON Étienne, « Michel Menot et la technique du sermon médiéval », dans Id., Les idées et les lettres, Paris, Vrin, 1955.

GOYET Francis et NOILLE Christine, «Présentation générale », Exercices de rhétorique, n 1, 2013, en ligne sur <https://journals.openedition.org/rhetorique/87>. 
HAMESSE Jacqueline, « La méthode de travail des reportateurs », dans Dal pulpito alla navata. La predicazione medievale nella sua recezione da parte degli ascoltatori (secc. XIII-XIV), Atti del Convegno internazionale di storia religiosa in memoria di Zelina Zafarana, Medioevo e Rinascimento (Annuario del Dipartimento di studi sul Medioevo e il Rinascimento dell'Università di Firenze, vol. III), Florence, Olschki, 1989, p. 51-67.

LAUSBERG Heinrich, Elementi di retorica, Bologne, il Mulino, 1969.

LEFEBVRE Charles, «Épikie », dans R. Naz (dir.), Dictionnaire de droit canonique, t. V, Paris, Letouzey \& Ané, 1953, ad vocem.

LEONARDI Claudio, "Jérôme Savonarole et le statut de la prophétie dans l'Église », Les textes prophétiques et la prophétie en Occident, Mélanges de l'École française de Rome, Moyen Âge, t. 102, $\mathrm{n}^{\circ} 2$, 1990, p. 589-596.

MARGEL Serge, «Le corps du témoin. Sur la vision eschatologique du martyr », Recherches de science religieuse, t. 102, $\mathrm{n}^{\circ}$ 3, 2014, p. 431-448.

Pellegrini Letizia, «La predicazione come strumento di accusa », dans Girolamo Savonarola. L'uomo e il frate, Atti del XXXV Convegno storico internazionale (Todi, 11-14 octobre 1998), Spoleto, Centro italiano di studi sull'alto Medioevo, 1999, p. 161-189.

Perelman Chaïm et OlBRETCHTS-TyteCA Lucie, Traité de l'argumentation. La nouvelle rhétorique, Bruxelles, Éditions de l'Université de Bruxelles, $2008^{6}$.

RÉGENT-SENSINI Anne, L'éloquence de la chaire. Les sermons de saint Augustin à nos jours, Paris, Seuil, 2009.

RIDolfI Roberto, Vita di Girolamo Savonarola, 6éd. révisée, Florence, Sansoni, 1981.

RUSCONI Roberto, «Reportatio ", dans Dal pulpito alla navata. La predicazione medievale nella sua recezione da parte degli ascoltatori (secc. XIII-XIV), Atti del Convegno internazionale di storia religiosa in memoria di Zelina Zafarana, Medioevo e Rinascimento (Annuario del Dipartimento di studi sul Medioevo e il Rinascimento dell'Università di Firenze, vol. III), Florence, Olschki, 1989, p. 7-36.

SAVonARola Girolamo, Prediche sopra Ezechiele, R. Ridolfi (éd.), Rome, Angelo Belardetti, 1955.

Savonarola Girolamo, Prediche sopra l'Esodo, P. G. Ricci (éd.), Rome, Angelo Belardetti, 1955.

Savonarola Girolamo, Prediche sopra Aggeo, L. Firpo (éd.), Rome, Angelo Belardetti, 1965.

Savonarola Girolamo, Prediche sopra Amos e Zaccaria, 3 vol., P. Ghiglieri (éd.), Rome, Angelo Belardetti, 1971.

Savonarola Girolamo, Prediche sopra i Salmi, 2 vol., V. Romano (éd.), Rome, Angelo Belardetti, 1974.

SAVONAROLA Girolamo, Lettere e scritti e scritti apologetici, R. Ridolfi, V. Romano et A. Verde (éds), Rome, Angelo Belardettti, 1984.

SERVENTI Silvia, « La parole des prédicateurs. Indices d'oralité dans les reportationes dominicaines (XIV $-\mathrm{XV}^{\mathrm{e}}$ siècle) », Cahiers de recherches médiévales et humanistes, $\mathrm{n}^{\circ}$ 20, 2010, p. 281-282.

TERREAUX-SCOTTO Cécile, « La désobéissance de Savonarole au pape Alexandre VI : une contestation religieuse pour un projet politique ", dans A. Morini (dir.), Papes et Papauté : respect et contestation d'une autorité bifrons, Saint-Étienne, P.U.S.E., coll. « Voix d'ailleurs. Études italiennes », 2013, ebook. 
TERREAUX-SCOTTO Cécile, « "Uno cappello di sangue” : formes du martyre dans les sermons de Savonarole », dans I. Fernandes (dir.), Martyr et martyre : évolutions et variations dans la Chrétienté de l'Europe occidentale, du haut Moyen Âge au XVI siècle, Cahiers pourpres, à paraître en 2020.

TERREAUX-SCOTto Cécile, « Les mots et les gestes de Savonarole », dans C. Lucas-Fiorato et J.-L. Fournel (dir.), Des mots et des gestes aux XVI ${ }^{e}$-XVII ${ }^{e}$ siècles en Italie et en France, Laboratoire italien, à paraître en 2020.

VERDE Armando F., "Savonarola lettore e commentatore del testo sacro », dans G. C. Garfagnini (dir.), Una città e il suo profeta. Firenze di fronte al Savonarola, Florence, Sismel Edizioni del Galluzzo, 2001, p. 183-200.

VERWILGHEN Albert, « Rhétorique et prédication chez Augustin », Nouvelle revue théologique, t. 120, $\mathrm{n}^{\circ} 2,1998$, p. 233-247.

\section{NOTES}

1. É. Gilson, « Michel Menot et la technique du sermon médiéval », dans Id., Les idées et les lettres, Paris, Vrin, 1955, p. 98.

2. F. Goyet et C. Noille, «Présentation générale », Exercices de rhétorique, ${ }^{\circ} 1,2013$, en ligne sur $<$ https://journals.openedition.org/rhetorique/87>.

3. Cf. S. Serventi, «La parole des prédicateurs. Indices d'oralité dans les reportationes dominicaines ( $\mathrm{XIV}^{\mathrm{e}}-\mathrm{XV}^{\mathrm{e}}$ siècle)», Cahiers de recherches médiévales et humanistes, $\mathrm{n}^{\circ} 20,2010$, p. 281-282.

4. Sur la prise de notes et la fiabilité des sermons (en particulier chez Bernardin de Sienne), voir J. Hamesse, «La méthode de travail des reportateurs ", dans Dal pulpito alla navata. La predicazione medievale nella sua recezione da parte degli ascoltatori (secc. XIII-XIV), Atti del Convegno internazionale di storia religiosa in memoria di Zelina Zafarana, Medioevo e Rinascimento (Annuario del Dipartimento di studi sul Medioevo e il Rinascimento dell'Università di Firenze, vol. III), Florence, Olschki, 1989, p. 51-67 et dans le même volume R. Rusconi, "Reportatio ", p. 7-36.

5. Comme l'a bien montré S. Dall'Aglio, «'Faithful to the Spoken Word': Sermons from Orality to Writing in Early Modern Italy ", The Italianist, vol. 34, n 3, 2014, p. 470-473.

6. On trouvera des remarques et des indications bibliographiques en ce sens dans l'introduction de l'article de Michele Lodone dans ce même volume.

7. Cité par S. Dall'Aglio, art. cité, p. 465.

8. G. C. Garfagnini, «Ser Lorenzo Violi e le prediche del Savonarola ", dans Id., "Questa è la terra tua». Savonarola a Firenze, Florence, Sismel Edizioni del Galluzzo, 2000, p. 3-27 ; Id., « Lorenzo Violi e il Savonarola ", Introduzione à L. Violi, Le Giornate, G. C. Garfagnini (éd.), Florence, Leo S. Olschki, 1986, p. IX-LXI.

9. G. Savonarola, Lettere e scritti e scritti apologetici, R. Ridolfi, V. Romano et A. Verde (éds), Rome, Angelo Belardettti, 1984, p. 150.

10. Qu'il me soit permis de renvoyer sur ce point à $C$. Terreaux-Scotto, « Les mots et les gestes de Savonarole ", dans C. Lucas-Fiorato et J.-L. Fournel (dir.), Des mots et des gestes aux XVI ${ }^{e}-\mathrm{XVII}{ }^{e}$ siècles en Italie et en France, Laboratoire italien, à paraître en 2020.

11. G. Savonarola, Prediche sopra Amos e Zaccaria, vol. I, P. Ghiglieri (éd.), Rome, Angelo Belardetti, 1971, sermon I, p.12. Désormais les pages se rapportant à ce sermon seront indiquées entre parenthèses dans le corps du texte. 
12. H. Lausberg, Elementi di retorica, Bologne, il Mulino, 1969, § 433, p. 241. Sur la sermocinatio chez Bernardin de Sienne, on lira avec profit la contribution de Valentina Berardini dans ce même volume.

13. Accademia d'Oropa, Alessandro VI e Savonarola (Brevi e lettere), Turin, ITER, 1950, p. 81.

14. G. Savonarola, Prediche sopra i Salmi, vol. II, V. Romano (éd.), Rome, Angelo Belardetti, 1974, sermon XXVIII, p. 199.

15. L. Violi, ouvr. cité, p. 37-38.

16. C. Lefebvre, «Épikie », dans R. Naz (dir.), Dictionnaire de droit canonique, t. V, Paris, Letouzey \& Ané, 1953, p. 365-366 et 373.

17. Accademia d'Oropa, ouvr. cité, p. 61.

18. Sur la désobéissance de Savonarole au pape, voir C. Terreaux-Scotto, «La désobéissance de Savonarole au pape Alexandre VI: une contestation religieuse pour un projet politique ", dans A. Morini (dir.), Papes et Papauté: respect et contestation d'une autorité bifrons, Saint-Étienne, P.U.S.E., coll. « Voix d'ailleurs. Études italiennes », 2013, e-book.

19. A.-L. Connesson, «L'apologue dans la prédication de Savonarole (Prediche sopra Amos e Zaccaria, 1496) », Chroniques italiennes, $\mathrm{n}^{\text {os }} 2-3,2003$, p. 94.

20. A. Verwilghen, «Rhétorique et prédication chez Augustin », Nouvelle revue théologique, t. 120, $\mathrm{n}^{\circ} 2,1998$, p. 246.

21. J'ai suivi ici la grille d'analyse que Perelman donne de l'hypothèse argumentative:

C. Perelman et L. Olbretchts-Tyteca, Traité de l'argumentation. La nouvelle rhétorique, Bruxelles, Éditions de l'Université de Bruxelles, $2008^{6}$, p. 197.

22. A. Régent-Sensini, L'éloquence de la chaire. Les sermons de saint Augustin à nos jours, Paris, Seuil, 2009 , p. 15.

23. L. Pellegrini, «La predicazione come strumento di accusa », dans Girolamo Savonarola. L'uomo e il frate, Atti del XXXV Convegno storico internazionale (Todi, 11-14 octobre 1998), Spoleto, Centro italiano di studi sull'alto Medioevo, 1999, p. 161-189.

24. É. Gilson, art. cité, p. 101. Sur le "thème", voir T.-M. Charland, o. p., Artes proedicandi. Contribution à l'histoire de la rhétorique au Moyen Âge, Paris, Ottawa, J. Vrin, Institut d'études médiévales, 1936, p. 111-123.

25. « Moderne » par rapport à l'éloquence des Pères de l'Église, le sermon scholastique a prévalu à partir du XIII ${ }^{\mathrm{e}}$ siècle. Codifié par les artes proedicandi, il est articulé autour du commentaire de l'Écriture, dont un passage - le «thème "- est expliqué en suivant plusieurs ramifications successives, l'ensemble du sermon étant assimilé à un arbre.

26. É. Gilson, art. cité, p. 120.

27. T.-M. Charland, ouvr. cité, p. 112-113, 122 et 194.

28. "Comme l'argile est dans la main du potier, Ainsi vous êtes dans ma main », Jérémie, 18:5-6. Voir aussi 2 Timothée, 2:20.

29. Cf. Romains, 9:21.

30. A. Régent-Sensini, ouvr. cité, p. 28.

31. Sur la prophétie chez Savonarole, voir au moins C. Leonardi, «Jérôme Savonarole et le statut de la prophétie dans l'Église ", dans Les textes prophétiques et la prophétie en Occident, Mélanges de l'École française de Rome, Moyen Âge, t. 102, nº 2, 1990, p. 589-596 ; J.-L. Fournel, « Les temps de la prophétie dans la Florence savonarolienne (automne 1494-été 1495) », dans A. Redondo (dir.), La prophétie comme arme de guerre des pouvoirs ( $\mathrm{XV}^{e}$-XVII ${ }^{e}$ siècles), Paris, Presses de la Sorbonne Nouvelle, 2000, p. 191-202 ; G. C. Garfagnini, « Alle origini dell'impegno politico savonaroliano: la profezia », dans Id., Questa è la terra tua, ouvr. cité, p. 431-439 et dans le même volume, Id., « Savonarole et la profezia: tra mito e storia », p. 29-55.

32. Cf. la définition qu'en donne Étienne Gilson : «Le prédicateur qui ne trouve pas dans un seul texte de quoi nourrir son développement peut en appeler d'autres au secours, à cette seule condition qu'ils s'accordent avec le premier. Tantôt ils s'accorderont verbalement, c'est-à-dire, 
qu'un même mot, présent dans chacun de ces textes, en assurera l'accord. Tantôt, au contraire, aucun mot commun ne reliera ces textes, mais ils s'accorderont pour le sens; ou encore, l'un des textes exprimera d'une manière plus parfaite ce que l'autre exprimait moins fortement.» (É. Gilson, art. cité, p. 137.)

33. G. C. Garfagnini, « Dire tutto quello che nel libro si contiene. La Bibbia di Savonarola », Archa Verbi, vol. 6, 2009, p. 124. Voir aussi L. Pellegrini, art. cité, p. 177.

34. Cité par A.F. Verde, "Savonarola lettore e commentatore del testo sacro", dans G.C. Garfagnini (dir.), Una città e il suo profeta. Firenze di fronte al Savonarola, Florence, Sismel Edizioni del Galluzzo, 2001, p. 185.

35. Cf. G. Savonarola, Prediche sopra i Salmi, ouvr. cité, vol. II, sermon XxvIII, p. 194-195.

36. G. Savonarola, Prediche sopra Amos, ouvr. cité, vol. III, sermon XLIII, p. 214.

37. Cité par R. Ridolfi, Vita di Girolamo Savonarola, $6^{\mathrm{e}}$ éd. révisée, Florence, Sansoni, 1981, p. 500.

38. Le rôle de la mémoire dans ces enchaînements est bien sûr fondamental, par les associations qu'elle crée.

39. Cette hypothèse est au cœur du volume que je suis en train de rédiger, L'édifice des sermons savonaroliens : rhétorique et politique à Florence à la fin $\mathrm{du} \mathrm{XV}^{e}$ siècle.

40. G. Savonarola, Prediche sopra l'Esodo, P. G. Ricci (éd.), Rome, Angelo Belardetti, 1955, p. 100.

41. G. Savonarola, Prediche sopra Amos, ouvr. cité, vol. I, sermon v, p. 85.

42. Id., Prediche sopra Ezechiele, R. Ridolfi (éd.), Rome, Angelo Belardetti, 1955, vol. I, sermon VI, p. 69.

43. S. Margel, «Le corps du témoin. Sur la vision eschatologique du martyr », Recherches de science religieuse, t. 102, $\mathrm{n}^{\circ} 3,2014$, p. 443. Sur la question du martyre chez Savonarole, voir C. TerreauxScotto, "Uno cappello di sangue" : formes du martyre dans les sermons de Savonarole", dans I. Fernandes (dir.), Martyr et martyre : évolutions et variations dans la Chrétienté de l'Europe occidentale, du haut Moyen Âge au XVI ${ }^{e}$ siècle, Cahiers pourpres, à paraître en 2020.

44. R. Ridolfi, Vita di Girolamo Savonarola, ouvr. cité, p. 24.

45. J. L. Austin, Quand dire, c'est faire, Paris, Seuil, 1970.

46. G. Savonarola, Prediche sopra Aggeo, L. Firpo (éd.), Rome, Angelo Belardetti, 1965, sermon XXI, p. 381.

\section{RÉSUMÉS}

À partir de l'hypothèse selon laquelle Savonarole affirme ne pas suivre les règles de composition des sermons préconisées par les artes prcedicandi pour au contraire mieux les exploiter, cet article examine l'ethos rhétorique du prédicateur. Il s'agit de montrer comment l'élaboration d'un plaidoyer prodomo - rendu nécessaire par les circonstances - sert les objectifs politiques du dominicain, pour qui l'action rhétorique et l'action politique sont une seule et même chose. Cette contribution montre également que Savonarole, contrairement aux règles du sermo modernus, ne bâtit pas ses sermons autour du commentaire d'un verset biblique mais privilégie un discours de ressassement articulé autour des temps présents.

Prendendo spunto dall'ipotesi secondo la quale Savonarola afferma di non conformarsi alle regole delle artes prcedicandi pur ispirandosi ad esse, questa contribuzione esamina l'ethos retorico del predicatore. Si tratta di mostrare che a nutrire gli obiettivi politici del domenicano, per il quale azione retorica e azione politica sono la stessa cosa, c'è la perorazione pro domo, necessaria 
date le circostanze. Questo contributo illustra anche che Savonarola, invece di seguire le regole del sermo modernus che impone di incentrare il discorso sul commento di un versetto biblico, preferisce elaborare un discorso ripetitivo di commento dei tempi presenti.

Starting from the hypothesis that Savonarole claims not to conform to the rules of the artes prcedicandi while drawing inspiration from them, this contribution examines the rhetorical ethos of the preacher. It is a matter of showing how the elaboration of a prodomo advocacy (made necessary by the circumstances) serves the political objectives of the Dominican, for whom the rhetorical action and the political action are the same thing. This contribution also shows that Savonarola, instead of following the rules of the sermo modernus-which requires focusing the discourse on the commentary of a biblical verse-, prefers to elaborate a repetitive commentary on the present times.

\section{INDEX}

Mots-clés : prédication, Savonarole, action rhétorique, action politique, dispositio

Parole chiave : predicazione, Savonarola, azione retorica, azione politica, dispositio

Keywords : preaching, Savonarola, rhetorical action, political action, dispositio

\section{AUTEUR}

\section{CÉCILE TERREAUX-SCOTTO}

Université Grenoble Alpes, LUHCIE.

cecile.terreaux@univ-grenoble-alpes.fr 